\title{
La tecnología de producción de coque de horno alto ante el nuevo
} milenio

\author{
R. Álvarez", M.A. Díez", C. Barriocanal* y J.L.G. Cimadevilla* \\ Resumen Para el funcionamiento del Horno Alto el coque es imprescindible. Sin embargo, la \\ ausencia de construcción de nuevas plantas de coquización a lo largo de los últimos años ha \\ producido un envejecimiento de las infraestructuras existentes para la producción de coque \\ siderúrgico. De esta forma, el desafío al que se enfrenta la industria de coquización ante el \\ nuevo milenio es la modernización de las viejas estructuras de producción de coque. En \\ cuanto a los sistemas de coquización alternativos, en la Unión Europea se ha desarrollado \\ el reactor Jumbo o Single Chamber System (SCS); en EE.UU. se tiende a emplear hornos no \\ recuperadores basados en los primitivos hornos de colmena; y en Japón se está \\ desarrollando el proyecto SCOPE 21.
}

Palabras clave Tecnología de coquización. Coque de horno alto. Single Chamber System. SCOPE 21. Tecnología no recuperadora.

\section{Technology of blast furnace coke production in the new millennium}

\begin{abstract}
Coke is essential for the operation of a Blast Furnace. However, in recent years new coking plants have not been built, which have caused the ageing of the existing infrastructures for blast furnace coke production. So, the challenge which coking industry in the new millennium has to face up is the modernization of the old coke production structures. In relation to alternative coking systems, in the European Union the Jumbo reactor or "Single Chamber System" (SCS) has been developed, whereas in USA non-recovery ovens based on the primitive beehive ovens are used. In Japan the project SCOPE 21 is being developed.
\end{abstract}

Keywords

Coking technology. Blast furnace coke. Single Chamber System. SCOPE 21. Non-recovery technology.

\section{INTRODUCCIÓN}

El acero continúa siendo la base del desarrollo económico al comienzo de este milenio y la fabricación del mismo basada en la ruta del Horno Alto no parece tener alternativa competitiva ni en el presente ni en un futuro inmediato ${ }^{[1}{ }^{2}$. El coque es imprescindible para el funcionamiento del Horno Alto y en los últimos tiempos, ante la ausencia de construcción de nuevas coquerías, se ha producido un envejecimiento de las infraestructuras existentes para la producción de coque siderúrgico. De esta forma, el desafío al que se enfrenta la industria de coquización ante el nuevo milenio es la modernización de las viejas estructuras de producción de coque. Para ello, la posible solución a este problema podría encontrarse entre alguna de las tres alternativas siguientes:

- prolongar la vida de las viejas baterías de coque mediante trabajos de reparación adecuados para poder llegar a los 40 años de vida útil, período que parecía inalcanzable en el pasado.

- construir nuevas coquerías convencionales dotadas de los últimos adelantos disponibles.

- desarrollar sistemas de coquización alternativos.

Los sistemas alternativos más importantes, SCS (Single Chamber System), SCOPE 21 y no recuperadores (non recovery), se han revisado recientemente $^{[3 \text { y } 4]}$. En el presente trabajo se describen dichas alternativas, se comparan con la tecnología 
convencional y se detallan los últimos avances en el desarrollo de la tecnología convencional de producción de coque (sistema de multicámaras).

\section{TECNOLOGÍA CONVENCIONAL Y SCS}

Con ocasión del $1^{\text {er }}$ Congreso Internacional de Coquización, que tuvo lugar en Essen en 1987, se introdujo con el nombre de JCR (Jumbo Coking Reactor) el proceso de coquización de cámaras individuales (SCS).

Este nuevo sistema de coquización proponía: (a) en lugar de emplear la carga de carbón húmeda del proceso convencional, ésta se cargaría precalentada; (b) sustituir el sistema de coquización con multicámaras, por la coquización en reactores individuales, más fáciles de controlar y automatizar; (c) en lugar de absorber las posibles fuerzas desarrolladas durante la coquización a través de paredes de calentamiento flexibles, que afectan a los hornos adyacentes, en el reactor individual dichas fuerzas se absorberían a través de paredes de calentamiento rígidas y estables, soportadas horizontalmente; (d) reemplazar los canales de calentamiento utilizados para proporcionar calor a dos cámaras contiguas para cada canal, por un sistema de calentamiento propio para cada reactor, el cual se puede controlar independientemente de los otros reactores; (e) en vez del suministro global de calor a una batería de multicámaras, en un reactor único dicho aporte sería individual y proporcional a la demanda; (f) en lugar de utilizar y explotar la energía recuperada del coque caliente y del gas bruto, para producir electricidad por ejemplo, sería posible utilizarla para precalentar el carbón; y (g) finalmente, en lugar de recuperar los subproductos clásicos del proceso convencional, sería posible la alternativa de producir hidrógeno o gas de síntesis.

Asimismo, un problema fundamental al que se enfrentan las coquerías convencionales es el de poder cumplir las actuales leyes de protección del medio ambiente, las cuales son cada vez más restrictivas. La multiplicidad de operaciones de carga y descarga de los hornos, todos los días, es una fuente potencial de emisiones que requiere control y considerables gastos de mantenimiento, a los que hay que añadir los frentes de sellado de los hornos.

En la tabla I se puede ver, sobre la base de una producción de $2 \mathrm{Mt}$ de coque/año, el estado del arte de las plantas de coquización y la propuesta para el nuevo sistema tal como se presentó en el $1^{\text {er }}$ Congreso de Coquización en $1987^{[5]}$.

En 1991, se unieron 13 empresas europeas (Fig. 1) para fundar el European Cokemaking Technology Center (ECTC). El objetivo fundamental era el desarrollo del reactor individual (Jumbo/SCS) en combinación con el precalentamiento del carbón. El precalentamiento del carbón, previo al proceso de coquización en los hornos de coque, es una tecnología que se introdujo industrialmente al final de los años 70, con plantas instaladas en varios países, pero que desapareció a finales de los 90 . Esta tecnología ha sido descrita con detalle en publicaciones anteriores ${ }^{[6}$ y 7$]$. El proceso de precalentamiento seleccionado para el ECTC fue el Precarbon, del cual estuvo operando en el INCAR una planta piloto de $2 \mathrm{t} / \mathrm{h}$ desde finales de los 80 hasta comienzo de los $90^{[7 \mathrm{y} 8]}$.

El proyecto Jumbo/SCS obtuvo la vitola de proyecto Eureka, debido al número de participantes de distintos países europeos y la alta naturaleza tecnológica de la propuesta. En febrero de 1992

Tabla I. Evolución de las dimensiones de los hornos de coque y dimensiones de los hornos del sistema SCS

Table I. Evolution of the coke oven's dimensions and dimensions of the SCS ovens

\begin{tabular}{|c|c|c|c|c|c|c|}
\hline \multirow[t]{2}{*}{ DIMENSIONES } & \multicolumn{2}{|c|}{1970} & \multirow[t]{2}{*}{1980} & \multirow[t]{2}{*}{1990} & \multicolumn{2}{|c|}{ JUMBO } \\
\hline & 1 & II & & & Básico & Posible \\
\hline Longitud, m & 14,2 & 16,4 & 15,9 & 18,0 & 19,0 & 25,0 \\
\hline Altura, m & 6,0 & 7,65 & 7,1 & 7,63 & 10,0 & 12,5 \\
\hline Anchura, mm & 450 & 435 & 590 & 610 & 850 & 850 \\
\hline VOLUMEN ÚTIL, $\mathrm{m}^{3}$ & 36,4 & 52,2 & 62,3 & 78,9 & 150 & 255 \\
\hline PRODUCTIVIDAD, t/horno & 21,3 & 32 & 39,8 & 48,7 & 100,0 & 165,0 \\
\hline No de Hornos & 187 & 123 & 142 & 120 & 55 & 33 \\
\hline Ciclos de apertura/d & 2.056 & 1.368 & 1.242 & 1.035 & 110 & 66 \\
\hline No de Deshornados/d & 257 & 171 & 138 & 115 & 55 & 33 \\
\hline Longitud de frentes de sellado, $\mathrm{km} / \mathrm{d}$ & 9,5 & 7,2 & 6,0 & 5,3 & 2,4 & 1,8 \\
\hline
\end{tabular}




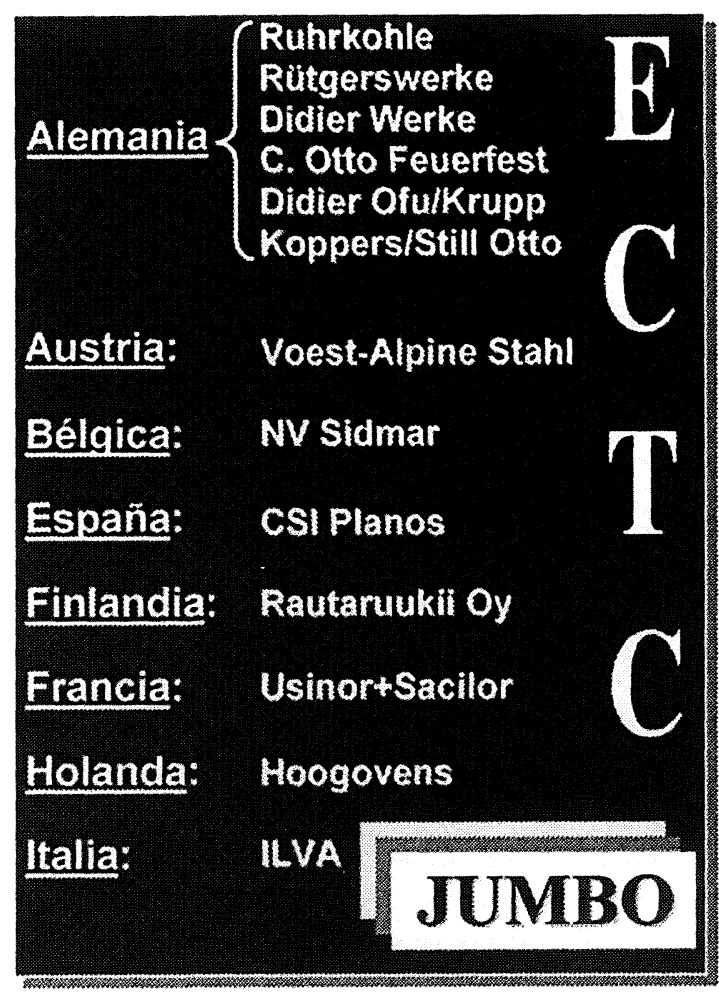

Figura 1. Empresas europeas fundadoras del European Cokemaking Technology Center (ECTC).

Figure 1. European companies founders of the European Cokemaking Technology Center (ECTC).

comenzó la construcción de una planta de demostración en Alemania y 12 meses más tarde comenzó el calentamiento del reactor. Se cargó el primer carbón y se deshornó el primer coque en abril de 1993. A partir de esta fecha comenzaron las pruebas, las cuales terminaron en febrero de $1996 . \mathrm{La}$ planta se desmanteló en el verano de 1998. Se han publicado muchos trabajos acerca del desarrollo y de los resultados de este proceso ${ }^{[9-15]}$, mencionándose incluso que el mismo ya estaba listo para su aplicación industrial ${ }^{[13]}$. En las figuras 2 y 3 , se resumen los datos más relevantes ${ }^{[15]}$ propuestos para el SCS, referentes al ahorro energético y a la calidad del coque.

Sin embargo, la realidad es que, a mediados del 2003, ni se ha construido ni está en fase de construcción ninguna planta SCS, a lo que hay que añadir que, además, en Alemania se ha seleccionado el sistema convencional, dotado de los últimos adelantos, para construir una nueva coquería.

\section{NUEVA COQUERÍA EN SCHWELGERN}

La planta más moderna del mundo hasta el siglo XXI -Kaiserthul (Alemania)- comenzó su funcio-

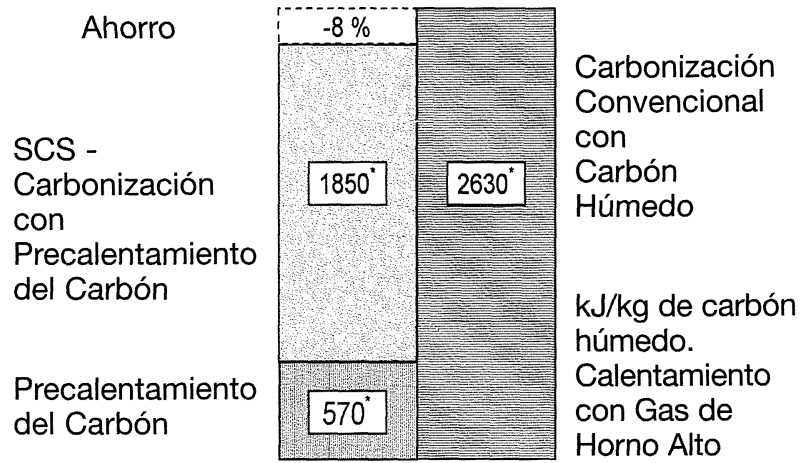

Figura 2. Ahorro energético mediante el proceso SCS.

Figure 2. Energy saving by SCS process.

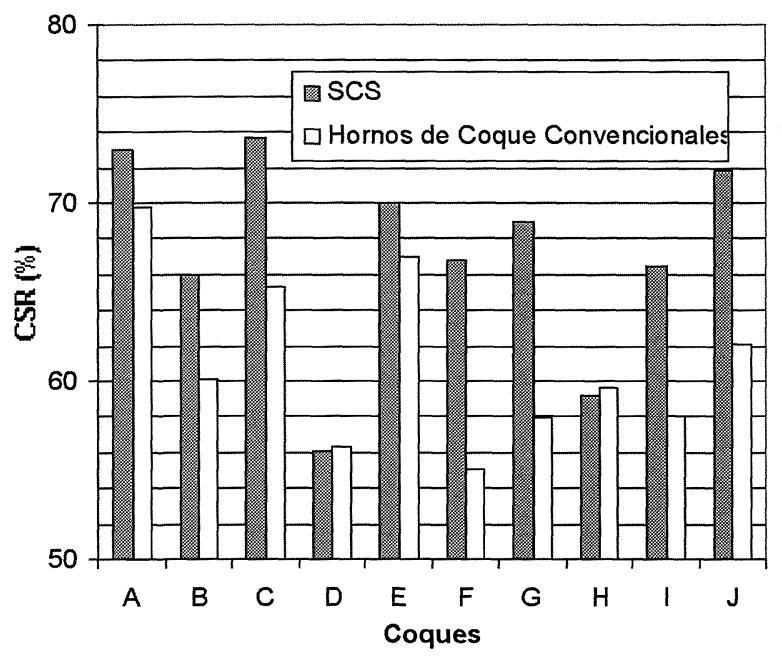

Figura 3. Resistencia post-reacción (CSR) de distintos coques.

Figure 3. Strength after reaction (CSR) of different cokes.

namiento en 1992 y se cerró en Diciembre de 2000, con menos de 10 años de actividad.

Thyssen Krupp Stahl ha construido ${ }^{[16]}$ la planta de coquización más moderna del mundo hasta el momento en Schwelgern, cerca de Duisburg (Alemania). Las razones para la construcción de esta coquería, que es la más grande del mundo, han sido publicadas ${ }^{[17]}$. Esta planta comenzó a funcionar, inicialmente por debajo de su capacidad máxima, en marzo de 2003. Las principales características de esta planta son las que se citan a continuación: 2 baterías de 70 hornos/batería, $20 \times 7,88 \times 0,59 \mathrm{~m}$ (longitud/altura/anchura), $93 \mathrm{~m}^{3}$ de volumen, $55 \mathrm{t}$ de coque/horno, 135 deshornados/día y 2,5 Mt de coque/año. 
Conviene destacar que, aunque inicialmente estaba pensado instalar un sistema de apagado en seco del coque, debido a la experiencia negativa de Kaisertuhl se ha instalado un sistema de apagado húmedo del coque desarrollado por la compañía, denominado CSQ (Coke Stabilization Quenching).

\section{HORNOS NO RECUPERADORES (Non recovery/heat recovery ovens)}

La llegada del Clean Air Act Amendment en 1990 marcó el comienzo de una nueva etapa en el proceso de coquización dentro de los EE.UU. El fuerte énfasis puesto en el control de las emisiones, combinado con el incremento de los costes de funcionamiento de los hornos de coque convencionales, con complicadas plantas de subproductos, dio lugar a que la industria siderúrgica de EE.UU. tratara de buscar tecnologías de coquización menos complejas y más aceptables desde el punto de vista ambiental. Así, la tecnología de coquización menos compleja era el primitivo horno de colmena, operativo desde el siglo XIX.

En el interior de estos hornos el carbón recibe el calor de la combustión directa del gas bruto. En este tipo de plantas se lleva a cabo la combustión de todo el gas producido, de forma que en el proceso se genera un importante excedente de energía, que es posteriormente explotada en una planta de cogeneración, aprovechando que el gas abandona el horno a una temperatura bastante elevada.

Existen varias plantas funcionando con este proceso $^{[18]}$, pero la más importante es la que tiene la compañía Indiana Harbor Coke cerca de Chicago y que está en funcionamiento ${ }^{[19]}$ desde 1998. En la figura 4 se muestra un diagrama general de dicha planta, cuyas características generales son: 268 hornos repartidos en 4 baterías, $14,3 \times 1,0 \times 3,7 \mathrm{~m}$ (longitud/altura/anchura), 41 t carbón/horno, $48 \mathrm{~h}$ de tiempo de coquización y 1,2 Mt coque/año.

Recientemente, se ha realizado una comparación ${ }^{[20]}$ entre estas dos tecnologías (convencional y no-recuperadora) en la que las principales conclusiones fueron:

- los beneficios para una planta con una producción de 1,3 Mt/año son similares en ambos casos;

- para una capacidad de producción mayor, de alrededor de $2 \mathrm{Mt}$ /año, la tecnología convencional parece resultar más ventajosa;

- si, por el contrario, la capacidad de producción está en el intervalo entre 500.000-700.000 t coque/año, la tecnología no recupera- dora parece más atractiva.

Sin embargo, a pesar de estas directrices generales, no resulta sencillo llegar a una elección, ya que hay otros factores que se deben tener en cuenta como, por ejemplo, las restricciones medioambientales o la disponibilidad de terreno.

\section{SCOPE 21 (Super Coke Oven for Productivity and Environmental enhancement towards the 21 st Century)}

Como alternativa al proceso de coquización convencional, Japan Iron and Steel Federation conjuntamente con Japan Center for Coal Utilization y con varias compañías siderúrgicas japonesas, están desarrollando un nuevo sistema de coquización

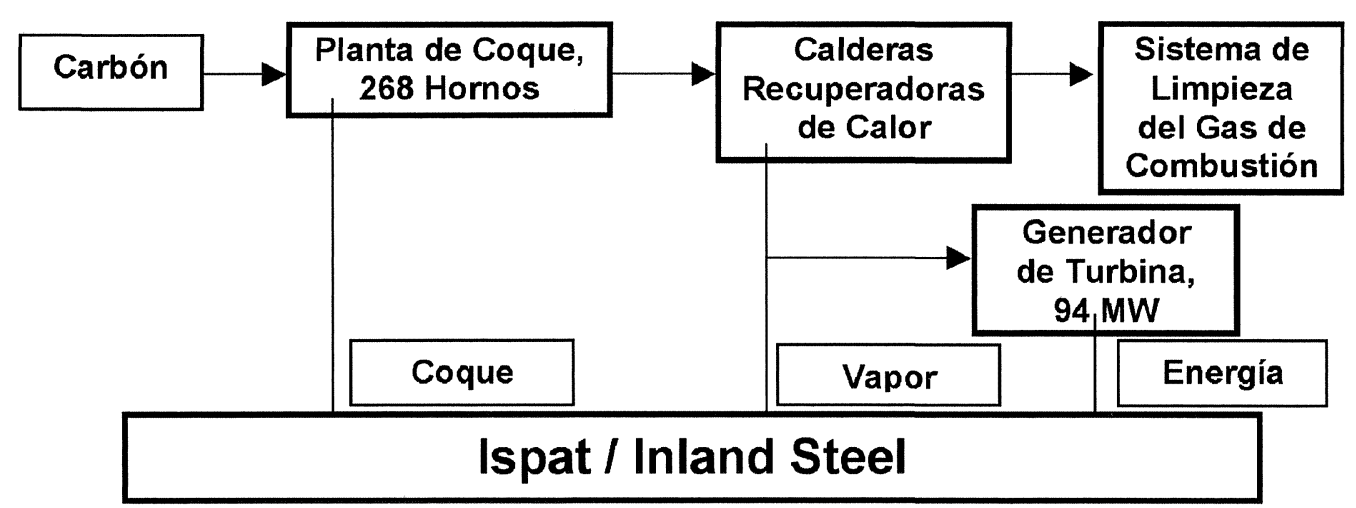

Figura 4. Diagrama general de una planta no recuperadora.

Figure 4. General diagram of a non-recovery plant. 
denominado SCOPE 21. Dicho proyecto comenzó en $1994^{[21-23]}$ y fue planeado inicialmente para tener un plazo de ejecución de 8 años. En este nuevo sistema de coquización (Fig. 5) el proceso se divide en tres etapas diferenciadas: pretratamiento de la mezcla de carbón, proceso de coquización a media temperatura y, finalmente, upgrading del coque seguido por el apagado en seco del mismo.

La primera etapa consiste en la separación de las partículas de carbón finas $(<0,3 \mathrm{~mm})$ y gruesas $(>0,3 \mathrm{~mm})$. Una vez separadas, se calientan rápidamente a una temperatura próxima a la de descomposición térmica del carbón $\left(350-450^{\circ} \mathrm{C}\right)$. El carbón fino se briquetea en caliente y se carga en un horno de coque convencional conjuntamente con el carbón grueso precalentado. La siguiente etapa es un proceso de coquización a una temperatura mucho más baja $\left(750-850^{\circ} \mathrm{C}\right)$ que en el proceso convencional. El coque se descarga del horno y se somete a un calentamiento posterior hasta $1.000^{\circ} \mathrm{C}$ en la parte superior de la instalación para apagado en seco del coque (CDQ).

La investigación a pequeña escala $(0,6 \mathrm{t} / \mathrm{h})$ ha finalizado y en estos momentos, con un año de retraso, están finalizando los ensayos en la planta piloto de $6 \mathrm{t} / \mathrm{h}$. A partir de aquí se decidirá la conveniencia de construir una planta comercial con la idea de producir $4.000 \mathrm{t}$ coque/d. Este proceso permite aumentar en las mezclas la cantidad de carbón no coquizable o poco coquizable, e incrementa considerablemente la productividad. Asimismo, dicho sistema conlleva ventajas adicionales en lo referente al control ambiental.

\section{CONCLUSIONES}

En lo relativo al estado actual y al futuro desarrollo de la producción de coque para H.A. se pueden establecer las siguientes conclusiones:

- las coquerías con hornos de coque convencionales y plantas de subproductos continúan siendo la base de la producción de coque en el mundo. La productividad de estos hornos se ha incrementado continuamente, alcanzando el máximo en los nuevos hornos de $93 \mathrm{~m}^{3}$ de la planta Schwelgern en Alemania;

- el control ambiental de las coquerías convencionales ha mejorado considerablemente en los últimos tiempos;

- la combustión directa del gas bruto con generación de vapor, puede iniciar un nuevo concepto de planta de coquización en el que sólo se producirían coque metalúrgico y energía;

- el beneficio de la utilización del gas de coquería es de gran importancia para la economía de una planta de coque. Nuevos conceptos, como la generación de energía en ciclo combinado o la combustión directa del gas bruto, pueden abrir nuevas posibilidades;

- la tecnología no recuperadora (heat recovery/ non recovery) se puede considerar técnicamente probada y lista para su aplicación industrial;

- dos nuevos procesos, SCS y SCOPE 21, todavía no han sido aplicados a escala industrial. El futuro dirá si estos procesos pueden competir con las tecnologías ya instaladas a escala industrial.

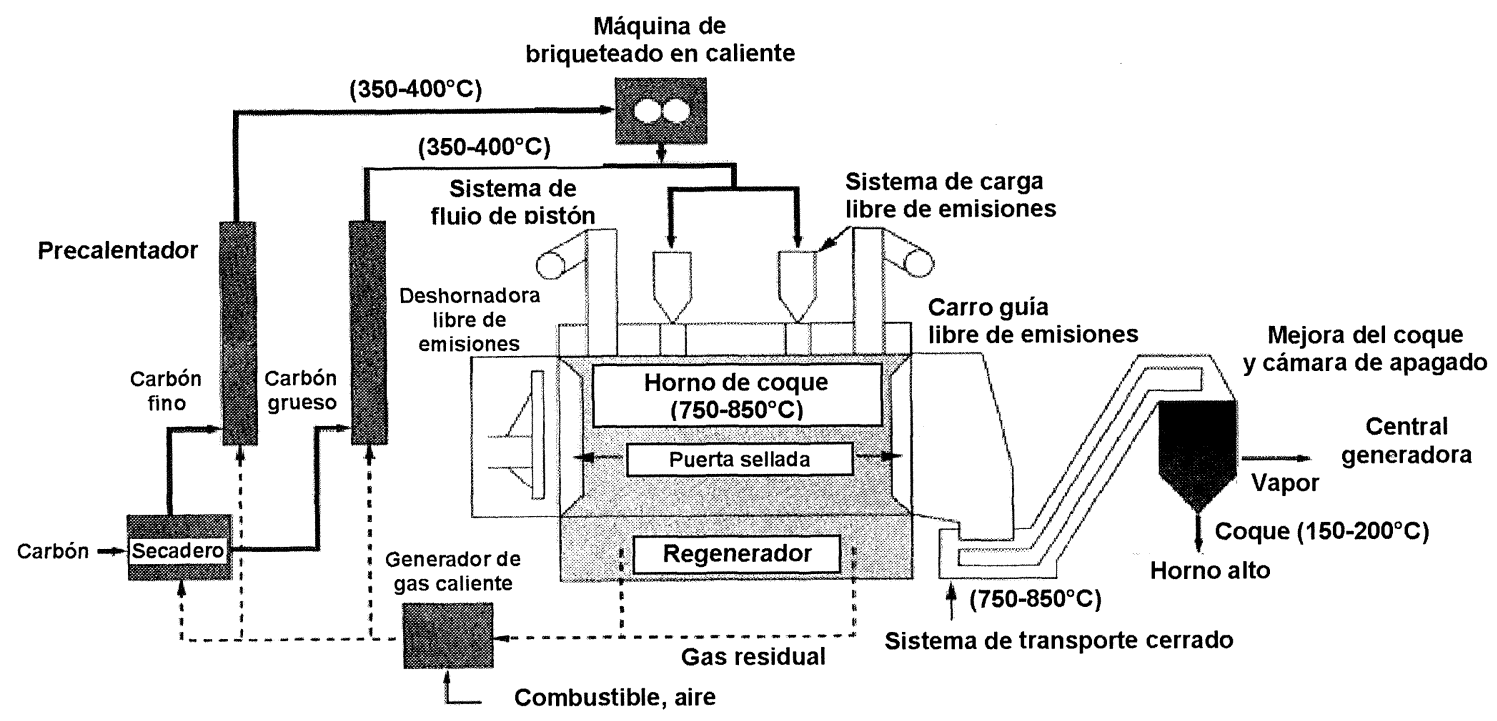

Figura 5. Proceso SCOPE 21.

Figure 5. Process SCOPE 21. 


\section{REFERENCIAS}

[1] G. NASHAN, Cokemaking Int. 2 (2001) 32-35.

[2] H.W. Gudenau, D. Senk, K. Fukada, A. Babich, C. Froehling, L.L. García, A. Formoso, F.J. Alguacil y A. CoRes, Rev. Metal. Madrid 39 (2003) 367-377.

[3] R. Álvarez, J.L. García-Cimadevilla, M.A. Díez, J. Bermúdez, V. Alonso y E. Fuente, Rev. Metal. Madrid 38 (2002) 380-387.

[4] M.A. Díez, R. Álvarez y C. Barriocanal, Int. J. Coal Geol. 50 (2002) 389-412.

[5] G. NASHAN, $1^{\text {st }}$ Int. Cokemaking Cong., Preprints vol. 1, Essen, Germany, 1987, Brinck \& Co KG, Essen, Alemania, 1987, A 2, pp. 1-51.

[6] R. Álvarez, Rev. Metal. Madrid 20 (1984) 278-287.

[7] M.A. Díez, R. Álvarez M. Sirgado y H. Marsh, ISIJ Int. 31 (1991) 449-457.

[8] R. Álvarez, E. Álvarez, C. SuÁrez, F. MÉndez De AndéS y M. Sirgado, Essen, Alemania, 1987, Brinck\&Co KG, E5, p 20.

[9] H. Bertling, W. Rohde y K. Wessiepe, $2^{\text {nd }}$ Int. Cokemaking Cong., Londres, Reino Unido, The Institute of Materials, 1992, vol.2, pp. 467-489.

[10] H. BeRTLING y W. ROHDE, Cokemaking Int. 2 (1995), 120 125.

[11] H. Bertling y W. ROHDE, Proc. $3^{\text {rd }}$ Int. Cokemaking Cong., Gent, Bélgica, CRM-VDEh, 1996, pp. 273-278.

[12] G. NASHAN, K. WesSIEPE y G. WInZER, Cokemaking Int. 2 (1998) 30-55.
[13] D. Ameling, H. Baer, H. Bertling y H. B. Lüngen, Cokemaking. Int. 1 (1999), 32-39.

[14] G. Nashan, W. Rohde, K. Wessiepe y G. Winzer, Proc. $4^{\text {th }}$ European Coke Ironmaking Cong., Paris, Francia, 2000, ATS, vol.2, pp. 646-653.

[15] H. BAER, Proc. $4^{\text {th }}$ Eur. Coke Ironmaking Cong., París, Francia, 2000, ATS, vol.2, pp. 659-663.

[16] H. HofHerR, P. Liszio y G. Still. Cokemaking Int. 1 (2000) 39-45.

[17] P. Liszio y H. HofHerr, IISI Semin. Coke, Bruselas, 2001, Sesión 4, pp. 21-43.

[18] H. Toll y R.Worberg, $1^{\text {st }}$ China Int. Coking Technology Coke Making Congress, 2002, Beijing, China, Metallurgical Council, pp. 239-250.

[19] R.W. WestbrooK y K.J. Schuett, Proc. $4^{\text {th }}$ European Coke Ironmaking Cong., París, Francia, 2000, ATS, vol.2, pp. 654-658.

[20] H. Toll y P. E. Dremer, IISI Semin. Coke, Bruselas, Septiembre 2001, Sesión 4, pp. 67-80.

[21] K. NishioKA, Proc. $3^{\text {rd }}$ Int. Cokemaking Cong., Gent, Bélgica, CRM-VDEh, 1996, pp. 285-290.

[22] Y. NAKASHIMA, Technical Exchange Session, IISI, Committee on Technology, Bruselas, 1999, pp. 16-29.

[23] H. TAKetomi, K. Nishioka, Y. Nakashima, S. Suyama y M. MatsuUra, Proc. $4^{\text {th }}$ Eur. Coke Ironmaking Cong., París, 2000, ATS, vol.2, pp. 640-645. 